\title{
Simulation in material flow design
}

\author{
J. P. Saranen \\ Helsinki University of Technology, TAI Research Centre \\ P.O.B 9555, FIN-02015 HUT, Finland \\ Tel: $+358-9-4514819$ \\ Fax: +358-9-4513665 \\ e-mail: juha.saranen@hut.fi
}

\begin{abstract}
The objective of this paper is to discuss the role of discrete event system simulation in designing manufacturing layouts based on material flows. It is proposed that simulation should be used continuously during a layout project rather than just applying it as a one-shot analysis tool. A green field layout project where the approach is applied is presented. It is concluded that when applied properly discrete event system simulation provides the layout planners with insight to the functionality of the proposed system, which hopefully results in better layouts.
\end{abstract}

Keywords

Discrete event simulation, Layout design, Material handling, Factory logistics

\section{INTRODUCTION}

According to Phillips (1997) and Cedarleaf (1994) the handling, storage and movement of materials make up the largest components of the cost of the materials purchased and used in factory processes. Some of the negative impacts of bad layouts are higher material handling costs, cycle and lead time delays, high WIP and poor equipment utilization. The fact that these higher costs are usually incurred for several years stresses the importance of layout design.

Prior to the development of computerized planning methods, which are able to deal directly with transport costs, the intensity of different types of material flows were measured by the Systematic Layout Planning method developed by Richard Muthers. In the method, flows are converted into equivalent units by taking into account material characteristics such as shape, size, value and fragility (Phillips, 1997). Later the SLP method has been converted to computerized form. Graphical layout software often includes the ability to manually move the nodes and have the 
links drawn automatically. Some software include heuristics which generate layouts from design skeletons.

With the increasing power of desktop computers, commercially-available dynamic simulation models have proven to be an effective analysis aid in designing manufacturing plants. Despite the descriptions of the structure of sound simulation project found in literature and reported cases on the use of simulation in investment planning, discussions on how simulation should be applied as a part of industrial investment projects are scarce.

\section{LAYOUT DESIGN}

Detailed descriptions of the structure of the planning of a new manufacturing plant layout can be found in Phillips (1997) and Quartermann (1998). Omitting the location selection, the manufacturing plant layout process can be illustrated as seen in Figure . A precondition for the process is the specification of the manufacturing strategy and material handling system to be used. The manufacturing strategy already determines the capacities of the production processes in the factory (Phillips, 1997).

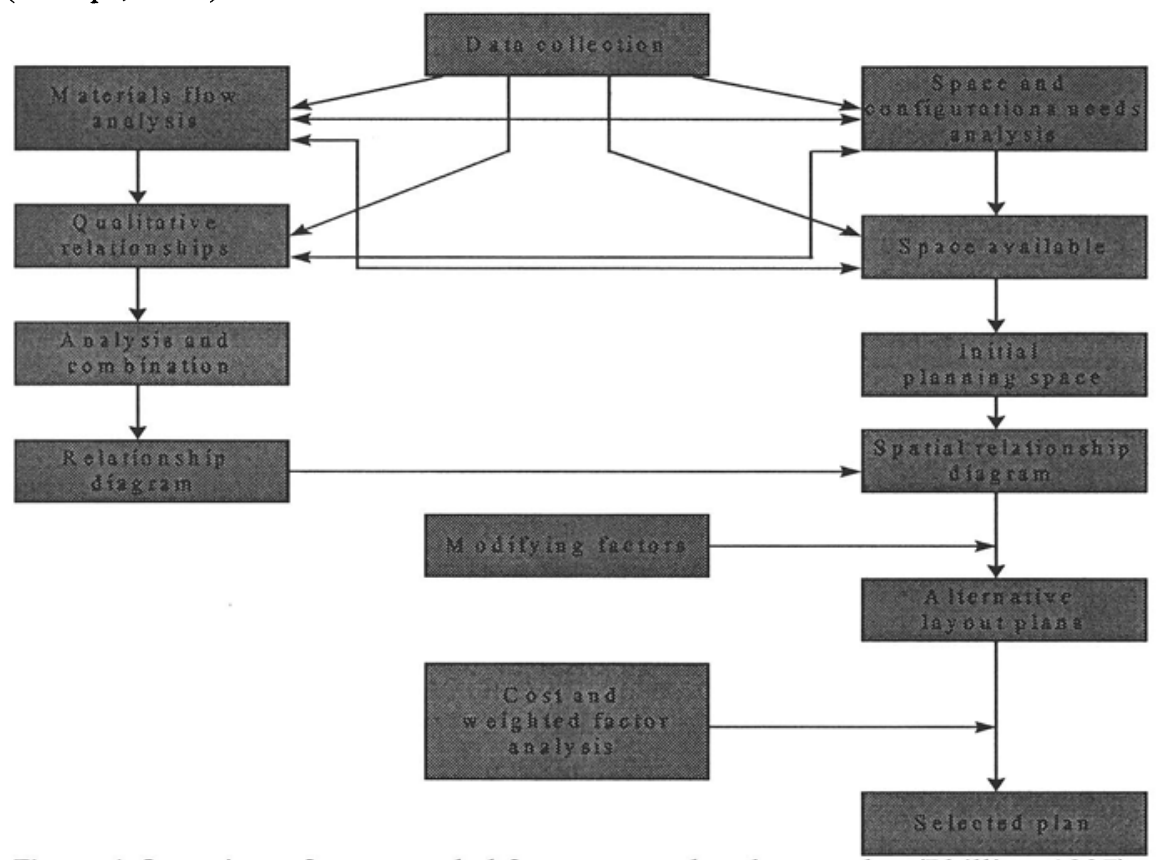

Figure 1 Overview of steps needed for a macro plant layout plan (Phillips, 1997)

Logically the procedure can be broken down into three tasks:

- determination of space needs of each production process, i.e. block

- determination of affinities between the blocks 
- developing layouts that fulfil the combination of the criteria above

Material flow analysis identifies the quantities of material to be moved between the blocks in the facility, based on production flow and capacities. Qualitative relationships are restrictions on the relative location of the blocks, they might be e.g. due to safety reasons or allowances for future space needs.

Using the distances and quantities in the spatial relationship diagram, respective material handling equipment need can be calculated, when the lot size to be moved and the velocity of the equipment is known. Whether this is done manually or using software, the calculations are static.

\section{DISCRETE EVENT SYSTEM SIMULATION}

Simulation is the imitation of the operation of a real world process or system over time (Banks et all, 1996). Naylor (1966) defines simulation as the process of designing a mathematical or logical model of a real system and then conducting computer-based experiments with the model to describe, explain, and predict the behavior of the real system.

In discrete-event simulation the state variables can change only at countable number of points in time (Law \& Kelton, 1991). Probabilistic models include at least one variable that is random. The output of a probabilistic model is also stochastic, and has to be treated as an estimate of the characteristics of the model. If the values of all the variables in the model are known exactly the output of the model is determined and the model is deterministic (Hoover \& Perry, 1989). In static simulation the model presents the system at one point of time. In dynamic simulation the model evolves over time (Law \& Kelton, 1991).

Simulation has several advantages compared to other modelling tools, according to Guedes (1995) simulation allows flexible and realistic modeling. Simulation also enables the study of non-existing systems (Hoover \& Perry, 1989).

Simulation analysis is a descriptive modeling technique. It does not provide explicit problem formulation and solution steps like linear programming. Descriptions of the structure of simulation analysis can be found in Banks et al. (1996), Law \& Kelton (1991) and Hoover \& Perry (1989).

Despite the descriptions of the structure of sound simulation project found in literature and reported cases on the use of simulation in investment planning, discussions on how simulation should be applied as a part of industrial investment projects are scarce. According to Banks \& Gibson (1998) designs evolve throughout a project life cycle for every industrial system project. Believing that changes to the facility or system design will not affect the system operation and therefore do not need to be included in the simulation model assumes knowledge that the analyst or project manager usually doesn't have. Therefore, to be valid and truly useful, a simulation model must be updated at each stage in a project's life cycle and the simulation experiments rerun to verify the new baseline. At a minimum, this should be done according to Banks \& Gibson (1998) at or near the end of each project phase. Artto et al. (1998) approach the subject from project 
management point of view. Simulation is seen as an effective planning tool for managing the end result of investment projects. According to the authors simulation should be applied through the planning project. The importance of simulation analysis culminates on the decision making points of the project. According to Artto et al. (1998) the strength of simulation lies in the fact that it can be applied already in the early project phases to support decisions where major project costs are fixed.

Simulation is not a universal problem solving tool. Banks \& Gibson (1997) list rules to determine whether simulation is the appropriate tool. Simulation is seen to be inappropriate, e.g., when the problem can be solved by using common sense analysis or analytically. If simulation is chosen to used, there are still several ways to fail. In addition to inaccuracies in 'mathematical correctness' of modelling the project can fail to have a well-defined set of objectives or it can fail due to communication problems with management (Law \& Kelton, 1991).

\section{THE CASE}

The case includes a study of a new facility investment project of a major Nordic food manufacturer. The aim of the investment was to construct a multi floor facility where all material is moved on pallets controlled and run by fixed automated equipment. According to the plans there is a monorail system on each floor. The monorails are connected to lifts by conveyors. The system supplier provided the cycle times as well as the preliminary structure of the monorailsystem, conveyors and lifts for the simulation modelling. The weekly production schedule was developed by the manufacturer.

The objective in the case is to find a block layout concept that minimizes the requirements of the fixed automated material handling equipment in the master facility plan, within the constraints given by the concurrent overall layout project. In addition to a block layout suggestion, this project results in

- an estimate for materials handling equipment needed and

- an evaluated control logic for the equipment.

Prior to simulation the system was analysed by using a static CAD-based spreadsheet model. The tentative analysis served as a conceptual model definition and data gathering phase for the simulation project. This tentative analysis also indicated that the scope suggested by the supplier for the materials handling system might include unnecessary capacity reservations. Two basic layout alternatives remained to be studied further by simulation analysis.

Because the simulation analysis was actualized as a part of a concurrent overall layout project, the information flow between the projects was essential for the simulation to be useful. The outline of the simulation project is presented in Figure . The subsequent layout alternatives and scenarios were defined in regular meetings with the client and overall layout planners as the project proceeded. This was necessary because the results of the milestones and the concurrent overall layout project affected the focus of the proceeding research. The gradual building of the 
simulation models in association with the client was beneficial because of the associated credibility aspects.

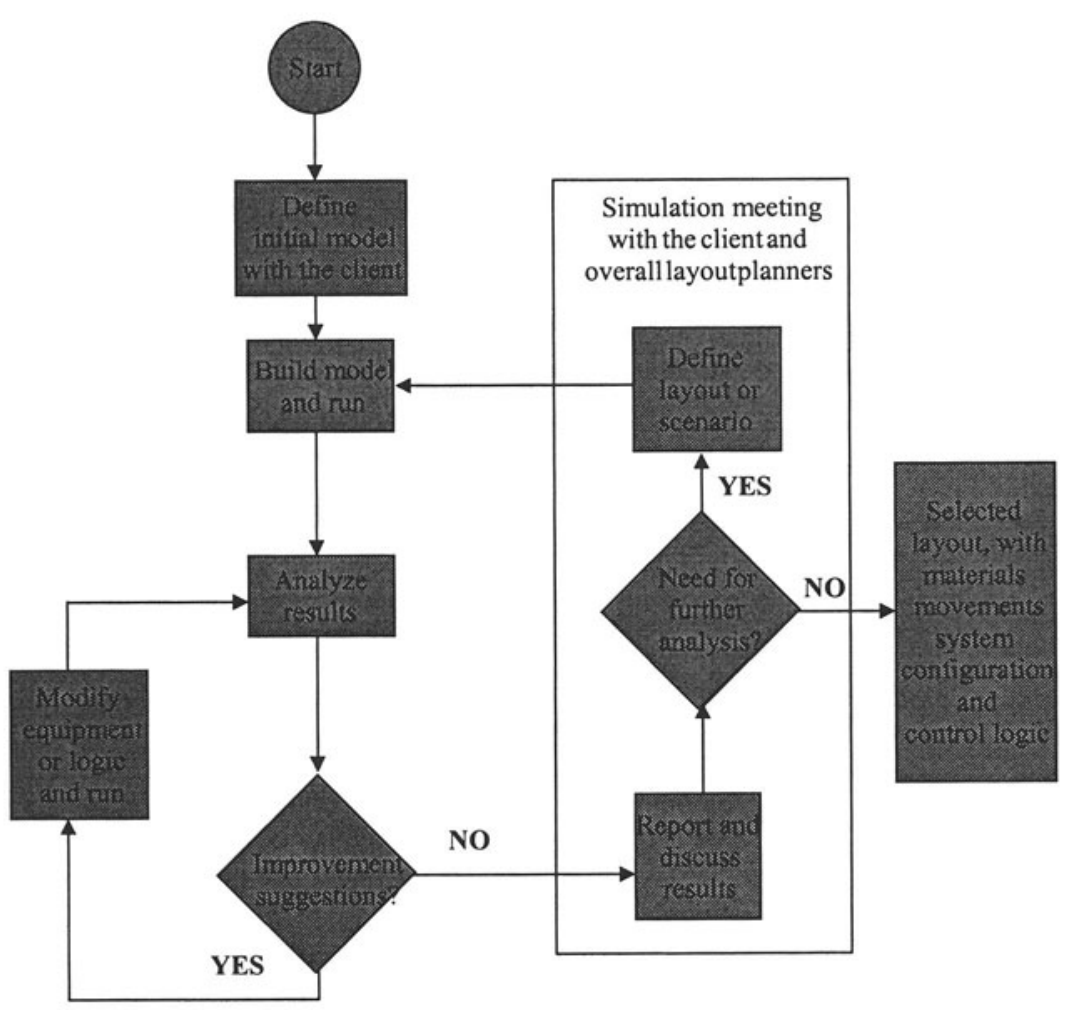

Figure 2: The outline of the simulation project

There were five simulation meetings in total. The first simulation models were based on the static cad-model, which could not discriminate between two basic layout alternatives. This is because the percentage of distance to be traveled empty by the equipment had to be estimated outside the model. The level of detail and inclusion of time in the simulation models, however allows the development and comparison of different control logics for the material handling equipment. In the first simulation models the pallets arriving at the lifts were served on first in -first out basis. This logic overloaded the lifts. By visually observing the 3D model, it was discovered that this was due to the fact that the two-pallet capacity lifts were moving too many single pallets. should only. The logic was changed so that the pallets were served only in pairs. This reduced the number of stops and distances travelled. Simulation confirmed that the increased waiting of the first pallet in the pair did not affect the production processes concerned. By using simulation the number lifts could be reduced by the worth of 300000 ECUs. The layout choice 
could be made based on the number of needed monorail vehicles, since the more efficient layout needed 35 percent less vehicles.

During the project, the co-ordination between the production processes could be fine tuned. As the overall layout project proceeded, more realistic estimates of the space requirements for the blocks allowed more activities to be located on one floor.

\section{CONCLUSIONS}

This paper presented a case where discrete event simulation was applied in a materials flow project. Because simulation requires extensive model building time, using it as a tool must be based on the superiority of the analysis compared to alternative methods.

An alternative tool would be static calculations, which were used in the case for initial analysis. This method is sufficient when a group of material handling resources is dedicated to moving material between two locations. If there are several sources and destinations the distance traveled by the materials handling equipment depends on the control used and on the relative volume of the flows served. The control not only affects the material handling equipment need but also the waiting time of the flows to be served and thus build up of queues. In the case presented queuing behavior must be accounted for in the layout but cannot be presented in a static model. Furthermore, in reality the length of the path along which load is carried depends on the overall utilisation of the system, i.e. the monorail vehicle chooses a longer path if its primary path is blocked. In part the absence of time patterns could be overcome by breaking the analysis into time frames during which the flows of material can be treated as constant. Even if the flows were constant, variation in processing times, differences in cycle times and batch sizes lead to queues.

The performance of the lifts and connected conveyors could be approximated by using queue theory. Analytic solutions for service times and average queues, however, require at least service or arrival times to be exponential (Law and Kelton, 1991). Furthermore, there are no analytic solutions for the maximum queues available.

Because simulation does not provide any solution search algorithm, there is the problem of screening out alternatives by making ad hoc assumptions about the behavior of the system. The alternatives to be evaluated are restricted by the creativity of the simulation modeler and the overall layout planners. This subjective component comes into play at the two decision-making points in Figure and dictates the quality of the solution derived.

It can be concluded that discrete event simulation offers a powerful tool in layout design. It remains the only alternative available when designing highly complex systems. Since the method requires extensive model building time and does not include any solution search algorithm, it is better suited for restricted problems where the alternatives to be evaluated are structurally similar. In layout planning 
simulation should be used continuously after the alternative layout plans have been defined. It is not only useful in evaluating alternatives, but also provides the planners a systems wide view which hopefully results in better layouts.

\section{REFERENCES}

Artto, K. A., Lehtonen, J-M \& Saranen, J., Managing Project Front-End Incorporating Strategic Early View to Project Management by Simulation, unpublished manuscript

Banks, J. Carson, J.S. \& Nelson, B. L., 1996, Discrete-Event System Simulation, Prentice-Hall, New Jersey

Banks, J. \& Gibson, R., 1997, 10 Rules for Determining when Simulation is Not Appropriate, IIE Solutions, September 1997

Banks, J. \& Gibson, R., 1998, Simulation Evolution, IIE Solutions, November 1998

Cedarleaf, J., 1994, Plant Layout and Flow Improvement, McGraw-Hill, USA.

Guedes, A. P., 1995, An Integrated Approach to Logistics Strategy Planning Using Visual Interactive Modelling and Decision Support, Ph. D. Thesis, School of Management, Centre for Logistics and Transportation, Cranfield University, UK

Hoover, S. \& Perry, R., 1989; Simulation - A problem solving approach, AddisonWesley, USA.

Law, A. M., \& Kelton , W., 1991, Simulation Modeling and Analysis, McGrawHill, USA.

Naylor, T. H., 1966, Computer Simulation Techniques, John Wiley and Sons, New York

Phillips E. J., 1997, Manufacturing Plant Layout - Fundamentals and fine points of optimum facility design, Society of Manufacturing Engineers, USA.

Quartermann, L., 1998, Facility Plannings Hidden Link, IIE Solutions, September 1998

\section{BIOGRAPHY}

Juha Saranen made his Master's Thesis in 1999 and is currently working at the TAI Research Centre in Helsinki University of Technology. His research interest areas are production systems, supply chains and discrete event simulation. 